\title{
Estimation of Stochastic Volatility with a Compensated Poisson Jump Using Quadratic Variation
}

\author{
Perpetual Saah Andam¹, Joseph Ackora-Prah ${ }^{1}$, Sure Mataramvura² \\ ${ }^{1}$ Department of Mathematics, Kwame Nkrumah University of Science and Technology (KNUST), Kumasi, Ghana \\ ${ }^{2}$ Department of Actuarial Science, University of Cape Town, Cape Town, South Africa \\ Email: p.andam@aims.edu.gh, jaackora-prah.cos@knust.edu.gh, Sure.Mataramvura@uct.ac.za
}

How to cite this paper: Andam, P.S., Ackora-Prah, J. and Mataramvura, S. (2017) Estimation of Stochastic Volatility with a Compensated Poisson Jump Using Quadratic Variation. Applied Mathematics, 8, 987-1000.

https://doi.org/10.4236/am.2017.87077

Received: June 9, 2017

Accepted: July 24, 2017

Published: July 27, 2017

Copyright (c) 2017 by authors and Scientific Research Publishing Inc. This work is licensed under the Creative Commons Attribution International License (CC BY 4.0).

http://creativecommons.org/licenses/by/4.0/

\begin{abstract}
The degree of variation of trading prices with respect to time is volatilitymeasured by the standard deviation of returns. We present the estimation of stochastic volatility from the stochastic differential equation for evenly spaced data. We indicate that, the price process is driven by a semi-martingale and the data are evenly spaced. The results of Malliavin and Mancino [1] are extended by adding a compensated poisson jump that uses a quadratic variation to calculate volatility. The volatility is computed from a daily data without assuming its functional form. Our result is well suited for financial market applications and in particular the analysis of high frequency data for the computation of volatility.
\end{abstract}

\section{Keywords}

Stochastic Volatility, Compensated Poisson Jump, Quadratic Variation

\section{Introduction}

Volatility is the downward and upward movements of the market. Naturally stock prices attract volatility. Economists argue that when demand is high, price goes up and producers are willing to sell more because a lot of people want to buy the same goods. Also higher supply leads to price fall and producers are willing to supply less because a lot of people do not want to pay more for goods that can be easily found in the market. The biggest driver of volatility is a drop in the market which later goes up for a while and comes down again. Mostly, when the economy is in a very precarious state, investors intervene to help solve the crisis which causes the stock market to revive and recover from its decline [2]. 
High volatility brings panic to investors and this fear has an impact on the financial market. Volatility of a financial instrument measures the uncertainty of the returns. It is an important parameter in pricing of an asset, portfolio management, investment analysis and risk management. Volatility influences cash flow from selling asset at a specific future date. Higher volatility means a greater chance of a fall in the price of an asset. Investors find it difficult to determine the movement of the prices of stock because of the stochastic nature of volatility which makes it difficult to predict stock prices. In 1973, Fischer Black and Myron Scholes published a paper called "The Pricing of Options and Corporate Liabilities" [3] in which volatility was assumed to be constant. In reality, real stock returns have higher kurtosis; there is a high chance of large price change. Empirical evidence has shown that, volatility assumed constant is restrictive, therefore estimation of volatility is the first step to adjust the Black-Scholes model to real data. Volatility cannot be directly observed and must therefore be estimated from historical data.

In most recent financial econometrics literature, volatility is regarded as a high degree of persistence and time-varying [4]. The development of new methods for estimating volatility still play active role in research. There is much evidence of volatility affecting financial assets and so estimating the ex-post volatility is an important tool for financial economics research. The common way to estimate volatility is by summing the squared returns. Although methods for volatility estimate work under some assumptions, it runs into a problem when the data exhibit some kinds of jumps in real life. Mostly since volatility cannot be observed, most researchers fit it in parametric econometrics models like Generalized Autoregressive Conditional Heteroscedasticity (GARCH) and its family [5]. They also compute implied volatilities using Black-Scholes for option pricing or compute the absolute returns or ex-post squared [6]. Daily data available on prices of asset which are speculative allows quadratic variation method to be used to measure the activities of returns in financial market. Most researchers have advocated for quadratic variation as a nonparametric method to estimate volatility of an asset. Most researches done on quadratic variation use tick-by-tick intra daily data, weekly or monthly data [6] [7] [8]. Quadratic variation controls micro-structure effect, it is consistent and feasible [9] and the error of realized volatility of its moment and asymptotic distribution has been studied [7]. For Pure Jump and mixed Jump-Diffusion Processes, it is approximately free of measurement error under general conditions [6]. Estimation of instantaneous volatility or spot volatility for high frequency data using quadratic variation is being researched recently and has been tested on some simulations [10] [11] and it has numerical derivative involved in it [12] [13] [14]. Quadratic variation have been used to estimate the instantaneous volatility of an asset price without jump diffusion process [1]. A proposed extension of a rolling sample variance with a continuous record asymptotic analysis of the use of quadratic variation for the estimation of the asset returns has been done [15]. The inte- 
grated volatility was treated as a continuous time stochastic process using a high frequency data and established the link between integrated volatility estimators and spot volatilities [15].

Unlike many other researches that has been done on quadratic variation, there has not been a consideration of the addition of compensated poisson jump to the stochastic differential equation model. In view of this, we are motivated to propose a quadratic variation as a nonparametric method for estimating instantaneous volatility from a stochastic differential equation model by including compensated poisson jump as an extension. This jump process has an intensity which is able to capture the steepness and skewness in volatility smiles for short-dated options. The presence of the jump will add more reality to financial market applications.

Section 2 reviews some mathematical preliminaries used in estimating volatility.

Section 3 presents the estimation of stochastic volatility using quadratic variation.

Section 4 presents the analysis of the results using data.

Section 5 concludes the work.

\section{Mathematical Preliminaries}

In this section, we review some mathematical theories which are used in estimating volatility.

Definition $1 A$ real valued process $X$ defined on the filtered probability space $\left(\Omega, \mathcal{F}, \mathcal{F}_{t}, \mathbb{P}\right), t \geq 0$ is called a semi-martingale if it can be decomposed in the form of

$$
X(t)=M(t)+A(t)
$$

where $M$ is a local martingale and $A$ is a càdlàg adapted process of locally bounded variation [16].

Definition 2 Suppose $(M, d)$ is a metric space, and $E \subseteq \mathbb{R}$, a function $f: E \rightarrow M$ is a càdlàg function if $f$ is right-continuous with left limits. That is for every $t \in E$,

- the left limit $f\left(t_{-}\right):=\lim _{s \uparrow t} f(s)$ exists and

- the right limit $f\left(t_{+}\right):=\lim _{s \downarrow t} f(s)$ exists and equals $f(t)$ [17].

Definition 3 A poisson process is a stochastic process $N_{t}, t \in \mathbb{R}_{+}$which has a jump size of 1 only and has a constant path between two jumps. At time, $t$, the value of $N_{t}$ is

$$
N_{t}=\sum_{j=1}^{\infty} I_{\left[T_{j}, \infty\right)}(t)
$$

where

$$
I_{\left[T_{j}, \infty\right)}(t)= \begin{cases}1 & \text { if } t \geq T_{j} \\ 0 & \text { if } 0 \leq t<T_{j} .\end{cases}
$$


$j \geq 1$ and $T_{j}$ is the increasing family of jump times of $N_{t}: \lim _{j \rightarrow \infty} T_{j}=+\infty$ where

$N_{t}$ satisfies:

- Independent increments: $\forall\left\{0 \leq t_{0}<t_{1}<\cdots<t_{n}, n \geq 1\right\}$ the random variables $N_{t_{1}}-N_{t_{0}}, \cdots, N_{t_{n}}-N_{t_{n-1}}$ are independent.

- Stationary increments: $N_{t+h}-N_{s+h}$ has the same distribution as $N_{t}-N_{s}$, $\forall h>0$ and $0 \leq s \leq t$. [18]

Definition 4 The compensated poisson process $M_{t}=N_{t}-\lambda t, t \in \mathbb{R}_{+}$is a martingale with respect to its own filtration $\mathcal{F}_{t} \quad$ [18].

Definition 5 Let $V_{t}$ be a real-valued stochastic process defined on a probability space $(\Omega, \mathcal{F}, \mathbb{P})$ and with time $t$ that ranges over non-negative real numbers then the pth variation is defined as,

$$
V_{t}=\lim _{\|\Pi\| \rightarrow 0} \sum_{k=1}^{n}\left|V_{t_{k}}-V_{t_{k-1}}\right|^{p}
$$

where $\Pi \in[0, t]$, and $\|\Pi\|$ is the norm of the partition $0=t_{0}<t_{1}<t_{2}<\cdots<t_{n}=t$ such that we have $\|\Pi\|=\max \left\{\left(t_{i}-t_{i-1}\right), \forall i=1, \cdots, n\right\}$ if the above sum converges [19].

This is true under certain conditions for example, $p=1$ defines the first variation or total variation process, for $p=2$, the $p$ th variation equals the quadratic variation if the sum converges. Also, it is a bounded variation if and only if for $p=1, V_{t}<+\infty$.

For a generalized Itô processes,

$$
X_{t}=X_{0}+\int_{0}^{t} \sigma_{s} \mathrm{~d} B_{s}+\int_{0}^{t} \mu_{s} \mathrm{~d} s,
$$

where $B$ is a Brownian motion, its quadratic variation is given by

$$
[X]_{t}=\int_{0}^{t} \sigma_{s}^{2} \mathrm{~d} s \quad[20] .
$$

The quadratic variation of a compensated poisson process $M_{t}=N_{t}-\lambda t$ is

$$
[M]_{t}=\sum_{s \leq t} \Delta M_{s}^{2}=N_{t}[21]
$$

\section{Stochastic Volatility Estimation}

A poisson process added to the stochastic differential equation with Brownian motion dependent embodies discontinuities in the returns on stock. This helps fit a better market data with regards to the reflection of the reality in the stock market and also accurately calculate volatility because it incorporates the effect of uncertainty over the jump size [22]. When there is a jump-diffusion process, it can describe the stock prices more accurately at the expense of making the market incomplete because jumps in stock prices cannot be hedged in traded securities. When the market is incomplete then the payoffs of the option cannot be replicated which will make it difficult to price the option [22]. In order to describe the stock prices more accurately, a compensated poisson jump is incorporated in a stochastic differential equation.

Suppose that the price process $p(t)$ of an asset follows the Itô process of a 
semi-martingale form:

$$
\mathrm{d} p(t)=\alpha(t, B) \mathrm{d} t+\sigma(t, B) \mathrm{d} B(t)+\mathrm{d} M(t),
$$

where $M(t)=N(t)-\lambda t$ and $N(t)$ is a poisson process with intensity $\lambda, \alpha$ is the drift, $\sigma$ is the volatility, time is represented by $t$ and the Brownian motion is $B$ then $\alpha$ and $\sigma$ are adapted to a random process satisfying the conditions in (QA) below;

$$
E\left[\int_{0}^{T}(\alpha(t))^{2} \mathrm{~d} t\right]<\infty
$$

(QA)

$$
E\left[\int_{0}^{T}(\sigma(t))^{2} \mathrm{~d} t\right]<\infty
$$

where $\alpha(t)=\alpha(t, B), \sigma(t)=\sigma(t, B)$ and it's bounded by $|\alpha|+|\sigma| \leq c$, where $c$ is a constant.

The time interval can be split into subsequent equal intervals which contain a certain number of observations. The volatility can be expressed in terms of its mean quadratic variation. From equation (1), $\alpha=0$ implies an efficient market [23]. The drift term makes no contribution to the quadratic variation process [24]. The theorem below is the main results of this paper which exhibits the estimation of volatility with a compensated poisson jump.

Lemma 6 For the time interval $[t, t+\varepsilon]$, where $\varepsilon$ denotes the interval between the two consecutive points, the volatility is constant in the interval and its value of observed quotations of the volatility is the mean quadratic variation:

$$
\sigma^{2}(t)=\lim _{\varepsilon \rightarrow 0^{+}} \mathbb{E}\left[\frac{\int_{t}^{t+\varepsilon} \sigma^{2}(s) \mathrm{d} s}{\varepsilon} \mid \mathcal{F}_{t}\right] .
$$

Proof.

$$
\lim _{\varepsilon \rightarrow 0^{+}} \mathbb{E}\left[\frac{\int_{t}^{t+\varepsilon} \sigma^{2}(s) \mathrm{d} s}{\varepsilon} \mid \mathcal{F}_{t}\right]=\lim _{\varepsilon \rightarrow 0^{+}} \frac{1}{\varepsilon} \mathbb{E}\left[\int_{t}^{t+\varepsilon} \sigma^{2}(s) \mathrm{d} s \mid \mathcal{F}_{t}\right] .
$$

Assuming $\sigma$ is càdlàg adapted process, if $\mathcal{F}_{t}$ is generated by the full observation of the price process, then using Fatou's Lemma we have,

$$
\begin{aligned}
& \lim _{\varepsilon \rightarrow 0^{+}} \frac{1}{\varepsilon} \mathbb{E}\left[\int_{t}^{t+\varepsilon} \sigma^{2}(s) \mathrm{d} s \mid \mathcal{F}_{t}\right] \\
& =\mathbb{E}\left[\lim _{\varepsilon \rightarrow 0^{+}} \frac{\int_{t}^{t+\varepsilon} \sigma^{2}(s) \mathrm{d} s}{\varepsilon} \mid \mathcal{F}_{t}\right]=\mathbb{E}\left[\lim _{\varepsilon \rightarrow 0^{+}} \frac{\frac{\mathrm{d}}{\mathrm{d} \varepsilon} \int_{t}^{t+\varepsilon} \sigma^{2}(s) \mathrm{d} s \mid \mathcal{F}_{t}}{\mathrm{~d}}(\varepsilon) \mid\right. \\
& =\mathbb{E}\left[\lim _{\varepsilon \rightarrow 0^{+}} \frac{\mathrm{d}}{\mathrm{d} \varepsilon} \int_{t}^{t+\varepsilon} \sigma^{2}(s) \mathrm{d} s \mid \mathcal{F}_{t}\right]=\mathbb{E}\left[\lim _{\varepsilon \rightarrow 0^{+}} \sigma^{2}(t+\varepsilon) \mid \mathcal{F}_{t}\right] \\
& =\mathbb{E}\left[\sigma^{2}(t) \mid \mathcal{F}_{t}\right]=\sigma^{2}(t) .
\end{aligned}
$$

Hence, 


$$
\lim _{\varepsilon \rightarrow 0^{+}} \mathbb{E}\left[\frac{\int_{t}^{t+\varepsilon} \sigma^{2}(s) \mathrm{d} s}{\varepsilon} \mid \mathcal{F}_{t}\right]=\sigma^{2}(t) .
$$

The expression in Lemma 6 shows the volatility process integrated over a given interval $[t, t+\varepsilon]$. This expressions gives the general case for obtaining $\sigma^{2}(t)$. On the other hand, $\sigma^{2}(t)$ can be estimated from a given model, for instance equation (1), as shown in the theorem below.

If volatility is estimated, it will help identify the type of financial model the stock price follows; whether it is geometric Brownian motion or OrnsteinUhlenbeck process and many more for the purpose of option pricing. This will also help investors to evaluate the rate of their investment risks. The theorem below is the volatility estimate based on Equation (1).

Theorem 7 Suppose that the price process, $p$, is given as $\mathrm{d} p(t)=\alpha(t, B) \mathrm{d} t+\sigma(t, B) \mathrm{d} B(t)+\mathrm{d} M(t)$, then its volatility, $\operatorname{Vol}(p)(t)$ is;

$$
\operatorname{Vol}(p)(t) \equiv \sigma^{2}(t)=\lim _{\varepsilon \rightarrow 0^{+}} \mathbb{E}\left[\frac{(p(t+\varepsilon)-p(t))^{2}-\Delta\left(\sum_{j=1}^{\infty} I_{\left[T_{j}, \infty\right)}(t)\right)}{\varepsilon} \mid \mathcal{F}_{t}\right]
$$

where $\mathbb{E}\left[\cdot \mid \mathcal{F}_{t}\right]$ denotes the conditional expectation operator with respect to the $\sigma$-field $\mathcal{F}_{t}$.

Proof. Given a price process $\mathrm{d} p(t)=\alpha(t, B) \mathrm{d} t+\sigma(t, B) \mathrm{d} B(t)+\mathrm{d} M(t)$, we estimate the volatility for evenly spaced time intervals using quadratic variation as follows:

$$
\begin{gathered}
\mathrm{d} p(t)=\alpha(t, B) \mathrm{d} t+\sigma(t, B) \mathrm{d} B(t)+\mathrm{d} M(t) \\
(\mathrm{d} p(t))^{2}=(\alpha(t, B) \mathrm{d} t+\sigma(t, B) \mathrm{d} B(t)+\mathrm{d} M(t))^{2} .
\end{gathered}
$$

Since $\left(\mathrm{d} B(t) \mathrm{d} t,(\mathrm{~d} t)^{2}\right) \rightarrow 0$ and $(\mathrm{d} B(t))^{2} \rightarrow \mathrm{d} t$ [25], and $M(t)$ is a martingale, it implies that $[\mathrm{d} M(t), \mathrm{d} t]=0,[\mathrm{~d} M(t), \mathrm{d} B(t)]=0$, and we obtain,

$$
\begin{aligned}
& (\mathrm{d} p(t))^{2}=\sigma^{2}(t) \mathrm{d} t+(\mathrm{d} M(t))^{2} \\
& (\mathrm{~d} p(t))^{2}-(\mathrm{d} M(t))^{2}=\sigma^{2}(t) \mathrm{d} t .
\end{aligned}
$$

If $(p, M):[t, t+\varepsilon] \rightarrow \mathbb{R}$, then integrating Equation (2) with respect to the interval gives:

$$
\begin{gathered}
\int_{t}^{t+\varepsilon}(\mathrm{d} p(s))^{2}-\int_{t}^{t+\varepsilon}(\mathrm{d} M(s))^{2}=\int_{t}^{t+\varepsilon} \sigma^{2}(s) \mathrm{d} s \\
\int_{t}^{t+\varepsilon} \mathrm{d} p(s) \cdot \mathrm{d} p(s)-\int_{t}^{t+\varepsilon} \mathrm{d} M(s) \cdot \mathrm{d} M(s)=\int_{t}^{t+\varepsilon} \sigma^{2}(s) \mathrm{d} s .
\end{gathered}
$$

With respect to $p$ and $M$, we have their quadratic variation as $\mathrm{d} p(s) \cdot \mathrm{d} p(s)=\mathrm{d}[p, p]_{[s]}=\mathrm{d}[p]_{[s]}$ and $\mathrm{d} M(s) \cdot \mathrm{d} M(s)=\mathrm{d}[M, M]_{[s]}=\mathrm{d}[M]_{[s]}$, this implies that,

$$
\mathrm{d}[M]_{[s]}=\mathrm{d}[N-\lambda s, N-\lambda s]=\mathrm{d}\left(N_{s}-\lambda s\right)^{2}=\mathrm{d} N_{s} .
$$

Then we have, 


$$
\begin{gathered}
\int_{t}^{t+\varepsilon} \mathrm{d}[p]_{[s]}-\int_{t}^{t+\varepsilon} \mathrm{d}[M]_{[s]}=\int_{t}^{t+\varepsilon} \sigma^{2}(s) \mathrm{d} s \\
\int_{t}^{t+\varepsilon} \mathrm{d}[p]_{[s]}-\int_{t}^{t+\varepsilon} \mathrm{d} N_{s}=\int_{t}^{t+\varepsilon} \sigma^{2}(s) \mathrm{d} s \\
{[p]_{[t+\varepsilon, t]}-N_{[t+\varepsilon, t]}=\int_{t}^{t+\varepsilon} \sigma^{2}(s) \mathrm{d} s} \\
\left(p_{t+\varepsilon}-p_{t}\right)^{2}-\left(N_{t+\varepsilon}-N_{t}\right)=\int_{t}^{t+\varepsilon} \sigma^{2}(s) \mathrm{d} s .
\end{gathered}
$$

Dividing both sides by $\varepsilon$, we have,

$$
\frac{\left(p_{t+\varepsilon}-p_{t}\right)^{2}}{\varepsilon}-\frac{N_{t+\varepsilon}-N_{t}}{\varepsilon}=\frac{\int_{t}^{t+\varepsilon} \sigma^{2}(s) \mathrm{d} s}{\varepsilon} .
$$

Taking the conditional expectation of both sides we have,

$$
\begin{gathered}
\mathbb{E}\left[\left(\frac{(p(t+\varepsilon)-p(t))^{2}}{\varepsilon}-\frac{\left(N_{t+\varepsilon}-N_{t}\right)}{\varepsilon} \mid \mathcal{F}_{t}\right]=\mathbb{E}\left[\frac{\int_{t}^{t+\varepsilon} \sigma^{2}(s) \mathrm{d} s}{\varepsilon} \mid \mathcal{F}_{t}\right]\right. \\
\Rightarrow \lim _{\varepsilon \rightarrow 0^{+}} \mathbb{E}\left[\frac{(p(t+\varepsilon)-p(t))^{2}-\left(N_{t+\varepsilon}-N_{t}\right)}{\varepsilon} \mid \mathcal{F}_{t}\right]=\lim _{\varepsilon \rightarrow 0^{+}} \mathbb{E}\left[\frac{\int_{t}^{t+\varepsilon} \sigma^{2}(s) \mathrm{d} s}{\varepsilon} \mid \mathcal{F}_{t}\right] . \\
N_{t+\varepsilon}-N_{t} \text { can be written as } \Delta N_{t}, \text { but from Definition (3), } \\
N_{t}=\sum_{j=1}^{\infty} I_{\left[T_{j}, \infty\right)}(t), \text { then it follows that, } \\
\lim _{\varepsilon \rightarrow 0^{+}} \mathbb{E}\left[\frac{(p(t+\varepsilon)-p(t))^{2}-\Delta\left(\sum_{j=1}^{\infty} I_{\left[T_{j}, \infty\right)}(t)\right)}{\varepsilon} \mathcal{F}_{t}\right] \\
=\lim _{\varepsilon \rightarrow 0^{+}} \mathbb{E}\left[\frac{\int_{t}^{t+\varepsilon} \sigma^{2}(s) \mathrm{d} s}{\varepsilon} \mid \mathcal{F}_{t}\right] .
\end{gathered}
$$

From Lemma 6, we have,

$$
\sigma^{2}(t)=\lim _{\varepsilon \rightarrow 0^{+}} \mathbb{E}\left[\frac{\int_{t}^{t+\varepsilon} \sigma^{2}(s) \mathrm{d} s}{\varepsilon} \mid \mathcal{F}_{t}\right] .
$$

Therefore the volatility of a price process which has an evenly spaced interval is:

$$
\operatorname{Vol}(p)(t) \equiv \sigma^{2}(t)=\lim _{\varepsilon \rightarrow 0^{+}} \mathbb{E}\left[\frac{(p(t+\varepsilon)-p(t))^{2}-\Delta\left(\sum_{j=1}^{\infty} I_{\left[T_{j}, \infty\right)}(t)\right)}{\varepsilon} \mid \mathcal{F}_{t}\right]
$$

This operator $\left(\mathbb{E}\left[\cdot \mid \mathcal{F}_{t}\right]\right)$ is generated by the full observation of the price process until time $t$ where $\mathcal{F}_{t}$ is the observation of single path of the market evolution within a given period.

This theorem is a link to Lemma 6 , that is: 


$$
\begin{aligned}
\operatorname{Vol}(p)(t) & \equiv \sigma^{2}(t)=\lim _{\varepsilon \rightarrow 0^{+}} \mathbb{E}\left[\frac{\int_{t}^{t+\varepsilon} \sigma^{2}(s) \mathrm{d} s}{\varepsilon} \mid \mathcal{F}_{t}\right] \\
& =\lim _{\varepsilon \rightarrow 0^{+}} \mathbb{E}\left[\frac{(p(t+\varepsilon)-p(t))^{2}-\Delta\left(\sum_{j=1}^{\infty} I_{\left[T_{j}, \infty\right)}(t)\right)}{\varepsilon} \mid \mathcal{F}_{t}\right] .
\end{aligned}
$$

If the price process is independent of its past information, then $\operatorname{Vol}(p)(t) \equiv \sigma^{2}(t)$ will be,

$$
\sigma^{2}(t)=\lim _{\varepsilon \rightarrow 0^{+}} \mathbb{E}\left[\frac{(p(t+\varepsilon)-p(t))^{2}-\Delta\left(\sum_{j=1}^{\infty} I_{\left[T_{j}, \infty\right)}(t)\right)}{\varepsilon} \mid \mathcal{F}_{t}\right]=0
$$

since the martingale part of the price process will vanish and the jump is estimated from the price process and hence the volatility will be constant.

From Theorem 7, it can be seen that the compensated poisson jump has a negative effect on the price process. Also, Theorem 7 requires high frequency data since it is the time evolution of the price of an asset in its semi-martingale form with a compensated poisson jump. Theorem 7 can be further simplified to

$$
\operatorname{Vol}(p)(t) \equiv \sigma^{2}(t)=\lim _{\varepsilon \rightarrow 0^{+}} \mathbb{E}\left[\frac{(p(t+\varepsilon)-p(t))^{2}}{\varepsilon} \mid \mathcal{F}_{t}\right]-\lim _{\varepsilon \rightarrow 0^{+}} \frac{\Delta\left(\sum_{j=1}^{\infty} I_{\left[T_{j}, \infty\right)}(t)\right)}{\varepsilon}
$$

but it cannot be determined numerically, because $\mathbb{E}\left[\cdot \mid \mathcal{F}_{t}\right]$ cannot be deduced from the observations but it can be determined when the distribution of the price process is known. For example, if the distribution of the price process follows a normal distribution then, we can apply antithetic variate Monte Carlo simulation to it.

Antithetic variate is defined as;

$$
\begin{aligned}
& \frac{1}{\varepsilon} \int_{0}^{t}(p(s+\varepsilon)-p(s))^{2} \\
& =\frac{1}{n \varepsilon} \sum_{s=1}^{n}\left(\frac{(p(s+\varepsilon)-p(s))^{2}+\overline{(p(s+\varepsilon)-p(s))^{2}}}{2}\right)
\end{aligned}
$$

where $\overline{(p(s+\varepsilon)-p(s))^{2}}=(p(-s+\varepsilon)-p(-s))^{2}$, but

$p(s+\varepsilon)=p\left(s_{1}+\varepsilon, s_{2}+\varepsilon, \cdots, s_{n}+\varepsilon\right)$ and

$p(-s+\varepsilon)=p\left(-s_{1}+\varepsilon,-s_{2}+\varepsilon, \cdots,-s_{n}+\varepsilon\right)=p\left(1-s_{1}+\varepsilon, 1-s_{2}+\varepsilon, \cdots, 1-s_{n}+\varepsilon\right)$.

$s_{1}, s_{2}, \cdots, s_{n}$ are independent random numbers and are uniformly distributed on

$(0,1), 1-s_{1}, 1-s_{2}, \cdots, 1-s_{n}$ are also uniformly distributed on $(0,1)$ and it makes $(p(s+\varepsilon)-p(s))^{2}$ has the same distribution as $(p(-s+\varepsilon)-p(-s))^{2}$ since $1-s_{1}, 1-s_{2}, \cdots, 1-s_{n}$ are negatively correlated with $s_{1}, s_{2}, \cdots, s_{n}$.

Then $\mathbb{E}\left[\cdot \mid \mathcal{F}_{t}\right]$ can be computed as: 


$$
\mathbb{E}\left[\frac{(p(t+\varepsilon)-p(t))^{2}}{\varepsilon} \mid \mathcal{F}_{t}\right]=\frac{1}{\varepsilon} \int_{0}^{t}(p(s+\varepsilon)-p(s))^{2} .
$$

The algorithm below estimates $\mathbb{E}\left[\cdot \mid \mathcal{F}_{t}\right]$ using antithetic variate Monte Carlo simulation.

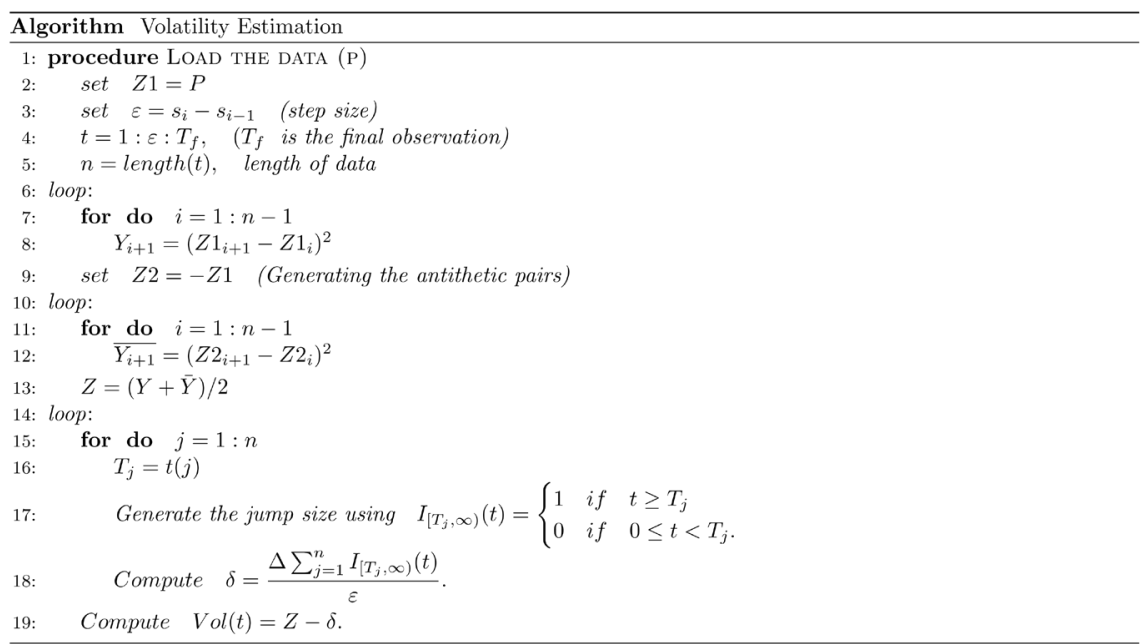

When simulating an evenly spaced data, there is a need to have a benchmark in order to assess the good prediction of the model proposed. To achieve this, we use the true volatility as the benchmark calculated as:

$$
\text { true volatility }\left(\sigma^{2}\right)=\sum_{t=1}^{n}(p(t+1)-p(t))^{2} .
$$

Equation (4) assists in assessing the performance of different estimators used. In this work, the estimators used are; the true volatility, the instantaneous volatility with a compensated poisson jump and the instantaneous volatility proposed by Malliavin and Mancino [1].

Malliavin and Mancino [1] proposed;

$$
\operatorname{Vol}(p)(t) \equiv \sigma^{2}(t)=\lim _{\varepsilon \rightarrow 0^{+}} \mathbb{E}\left[\frac{\int_{t}^{t+\varepsilon} \sigma^{2}(s) \mathrm{d} s}{\varepsilon} \mid \mathcal{F}_{t}\right]
$$

for calculating the instantaneous volatility without a jump. In order to apply data to Equation (5), we use antithetic variate Monte Carlo simulation to evaluate it.

From the Algorithm above, 1 to 8 is used to estimate the true volatility using Equation (4). The algorithm numbered 1 to 13 is used to generate the antithetic variate Monte Carlo simulation using Equation (5) and the numbered algorithm from 1 to 19 is used to compute the instantaneous volatility with a compensated poisson jump using Theorem 7 .

\section{Empirical Analysis of Theorem Using Data}

Stock prices indicate the strength and performance of the company. Any data can be used but with reference to this analysis we use data from AngloGold 
Ashanti Ghana (AGA). A daily high frequency stock prices data from AGA containing the closing prices from 16 April 2008 to 17 June 2016 was plotted with time (in years) as shown in Figure 1.

Closer to the later part of 2015 to 2016, the graph exhibits some sort of a jump. At the top of bear market, returns are earned than the bottom of a bull market. Increase in stock prices means investors are buying stocks rather than selling it and the fall in the price of stocks means investors are selling their stocks rather than buying. Also, an increase in the stock price of a company means there is a profit making and a risk reduction of layoff of workers in the company. When there is an increase in stock price, good returns are generated. When the stock price of a company falls continuously then there is a higher chance of a takeover by another company, a merge or even close down of the company.

The volatility is determined from the stock price of AngloGold Ashanti. It is used to determine the performance and strength of the stock price. It also help generate the investors return made from the stock price. The graphs below show the volatility of the stock prices obtained from AngloGold Ashanti.

The graph in Figure 2 indicates the plot of the true volatility using Equation (4). The graph in Figure 3 reveals the plot of the instantaneous volatility without a jump by Malliavin and Mancino (2009) [1] using Equation (5). The graph in Figure 4 shows the plot of the instantaneous volatility with a compensated poisson jump using Theorem 7 with the Algorithm stated above. The stock price is assumed to be normally distributed then antithetic variate Monte Carlo simulation is applied to it. The higher the volatility, the greater the returns and the higher the risk associated with the type of investment. Comparing the graphs in the stock prices as in Figure 1 and the volatility graphs in Figures 2-4, it shows that from 2009 to the mid of 2010, the stock prices increased sharply and its corresponding volatility was high which implies that investors were buying stocks rather than selling it. Also when there was a drastic decrease in stock prices from 2011 to 2014, the volatility also decreased sharply. Closer to 2015, the stock prices shot up again which made it highly volatile and might be

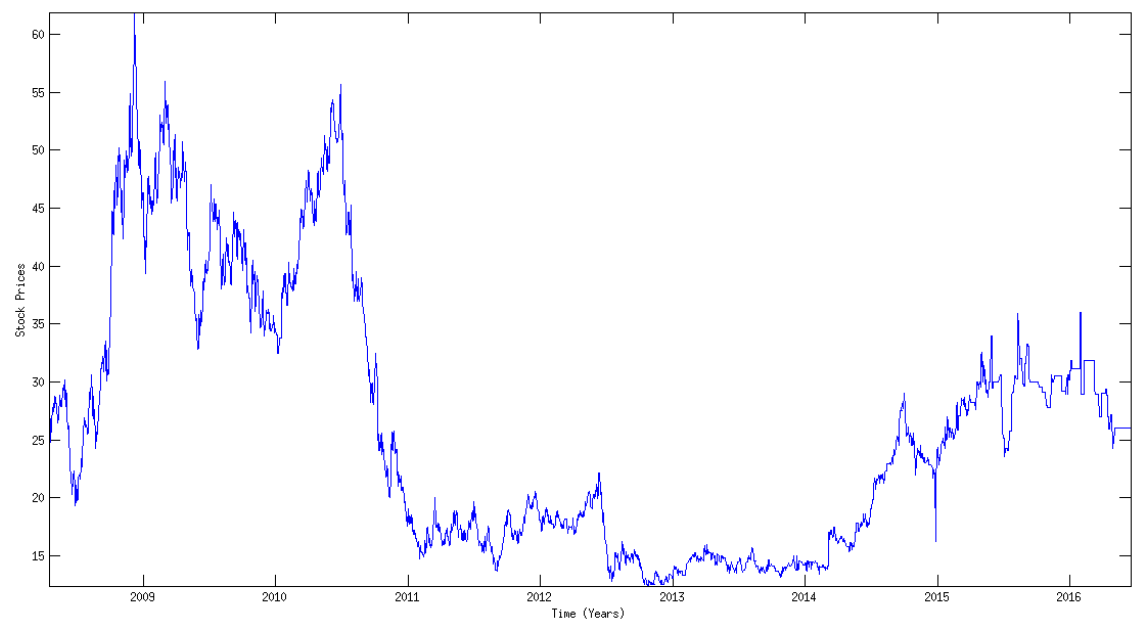

Figure 1. Plot of stock prices from AGA. 


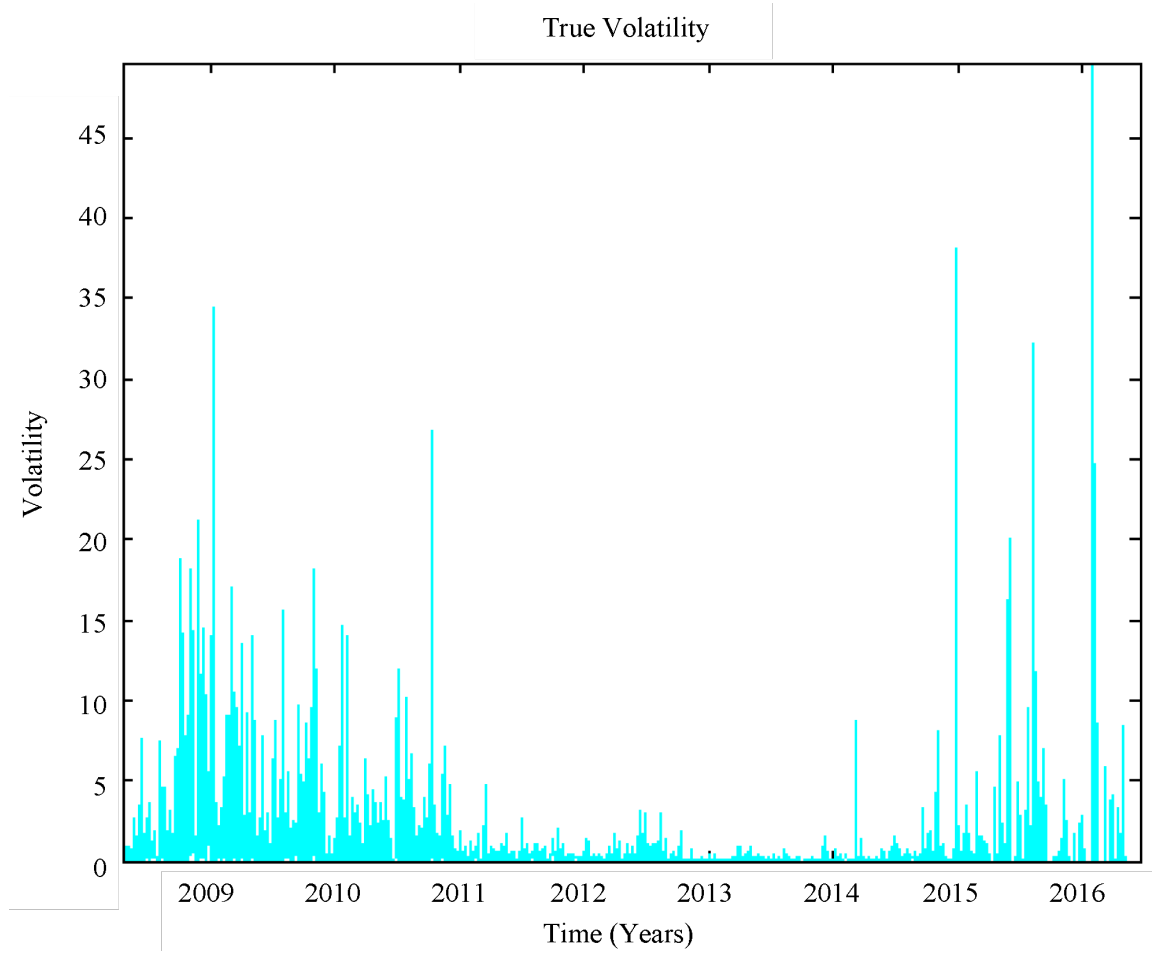

Figure 2. Plot of the True volatility.

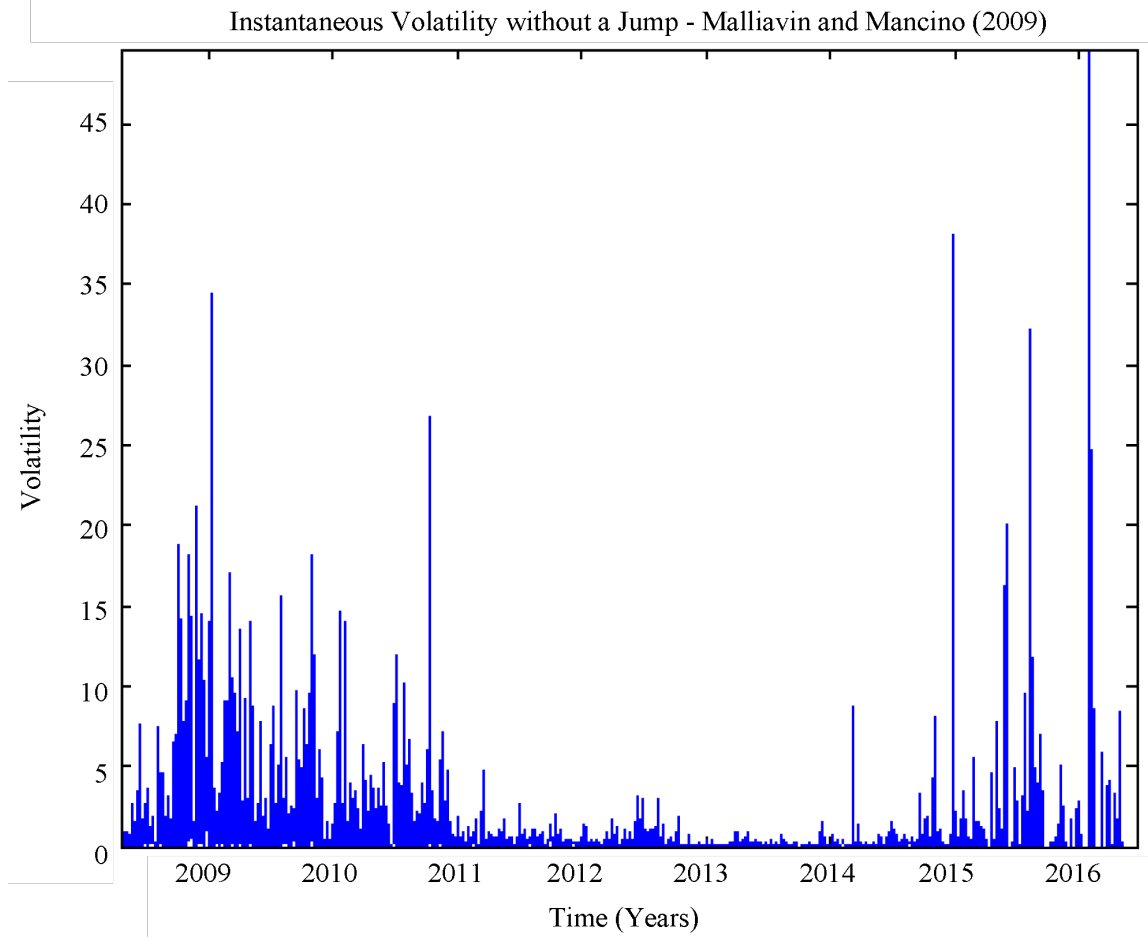

Figure 3. Plot of Instantaneous volatility without a jump Malliavin and Mancino (2009) [1].

attributed to the merge that took place between the then Ashanti Goldfields Corporation limited and AngloGold to form AngloGold Ashanti [26]. The trend of volatility helps the investor to re-balance its weights on portfolio of stocks and 


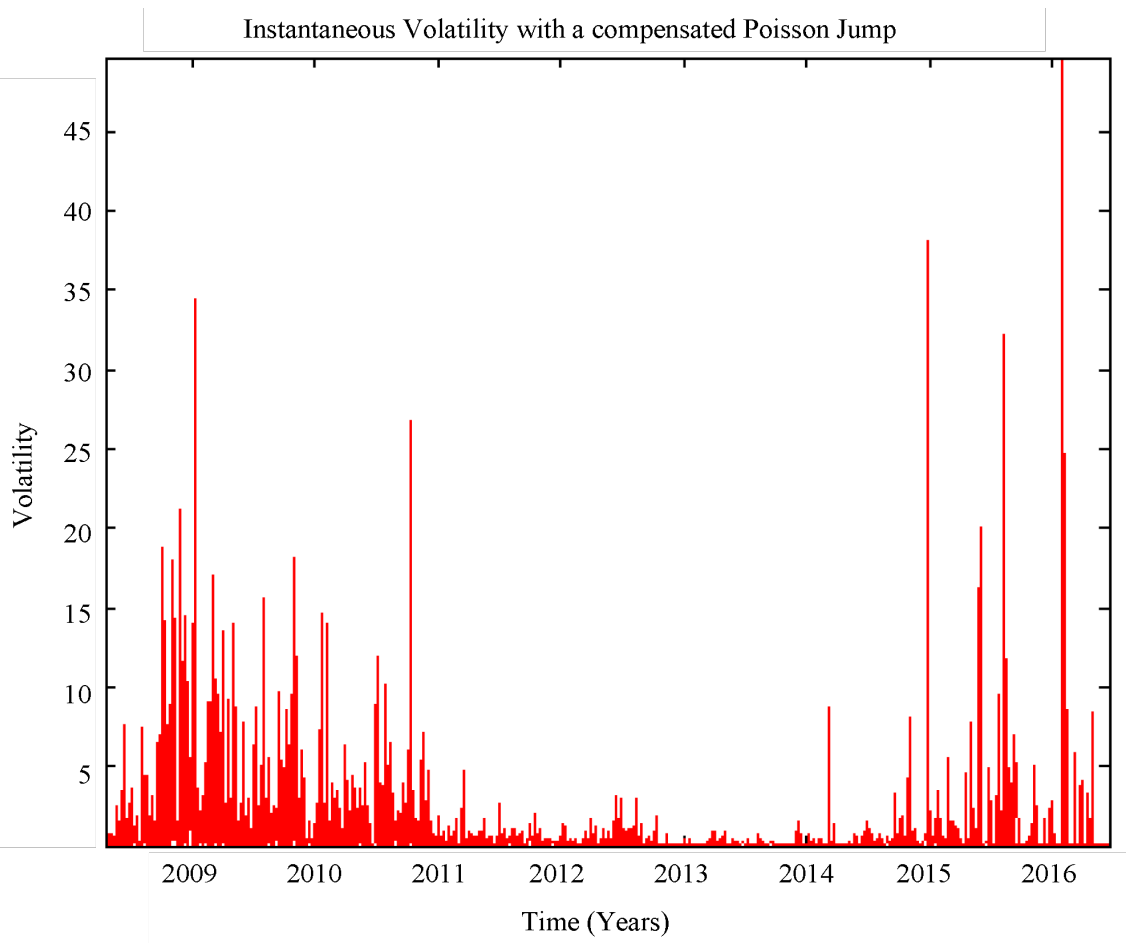

Figure 4. Plot of Instantaneous volatility with a compensated poisson jump.

also help the investor knows the performance and strength of the company.

\section{Comparison of Results}

The results obtained by Malliavin and Mancino [1] was

$$
\operatorname{Vol}(p)(t) \equiv \sigma^{2}(t)=\lim _{\varepsilon \rightarrow 0^{+}} \mathbb{E}\left[\frac{(p(t+\varepsilon)-p(t))^{2}}{\varepsilon} \mid \mathcal{F}_{t}\right]
$$

from the model $\mathrm{d} p(t)=\alpha(t, B) \mathrm{d} t+\sigma(t, B) \mathrm{d} B(t)$, and when compensated poisson jump was added to it we obtained

$$
\operatorname{Vol}(p)(t) \equiv \sigma^{2}(t)=\lim _{\varepsilon \rightarrow 0^{+}} \mathbb{E}\left[\frac{(p(t+\varepsilon)-p(t))^{2}-\Delta\left(\sum_{j=1}^{\infty} I_{\left[T_{j}, \infty\right)}(t)\right)}{\varepsilon} \mid \mathcal{F}_{t}\right]
$$

From this, it can be seen that the compensated poisson jump has an effect on the price process. Also, error analysis of the estimates were done using the Root Mean Square Error(RMSE) calculated as;

$$
\text { RMSE }=\sqrt{\frac{1}{N} \sum_{i=1}^{N}(\text { estimated }- \text { true volatility })^{2}} .
$$

The RMSE of the instantaneous volatility proposed by Malliavin and Mancino [1] was 3.0210 and the RMSE of the instantaneous volatility with a compensated poisson jump was 3.0184. This means that, the introduction of compensated poisson jump reduced the error margin and is a good predictive model than the 
instantaneous volatility proposed by Malliavin and Mancino [1]. This means that the stochastic differential equation with a compensated poisson jump is a better representative model for stock prices than stochastic differential equation without a compensated poisson jump.

\section{Conclusion}

We have established the theoretical basis for estimating stochastic volatility with the presence of a compensated poisson jump for univariate settings for evenly spaced observations using quadratic variation. The conditional expectation, $\mathbb{E}\left[\cdot \mid \mathcal{F}_{t}\right]$ was calculated using antithetic variate Monte Carlo simulation of the price process for implementation of data to be possible. We analyzed this using data from AGA, Ghana. Also, the RMSE of the instantaneous volatility with a compensated poisson jump gave a smaller value as compared to the RMSE of the instantaneous volatility proposed by Malliavin and Mancino [1]. It is therefore advisable for financial and economic analyst to employ jump processes when using such data for forecasting else; there will be error incorporated. This will help minimize the risk (in terms of loss) associated in investing in assets that are highly volatile like gold. It will be interesting to investigate the effect of other types of jumps on evenly spaced data.

\section{Acknowledgements}

This work was carried out with financial support from the government of Canada's International Development Research Centre (IDRC), and within the framework of AIMS Research for Africa Project and also our gratitude to the Department of Mathematics, KNUST, University of Cape Town and African Institute for Mathematical Science, Ghana.

\section{References}

[1] Malliavin, P. and Mancino, M.E. (2009) A Fourier Transform Method for Nonparametric Estimation of Multivariate Volatility. Annals of Statistics, 37, 1983-2010. https://doi.org/10.1214/08-AOS633

[2] Ibbotson, R.G. (2011) Why Does Market Volatility Matter? Yale School of Management, Yale School of Management.

[3] Black, F. and Scholes, M. (1973) Pricing of Options and Corporate Liabilities. Journal of Political Economy, 81, 637-654. https://doi.org/10.1086/260062

[4] Andersen, T.G., Bollerslev, T. and Diebold, F.X. (2002) Parametric and Nonparametric Volatility Measurement. NBER Technical Working Paper, 279.

[5] Barucci, E. and Reno, R. (2002) On Measuring Volatility and the GARCH Forecasting Performance. Journal of International Financial Markets, Institutions and Money, 12, 183-200.

[6] Andersen, T.G., Bollerslev, T., Diebold, F.X. and Labys, P. (2001) The Distribution of Realized Exchange Rate Volatility. Journal of the American Statistical Association, 96, 42-55.

[7] Barndorff-Nielsen, O.E. and Shephard, N. (2002) Econometric Analysis of Realized Volatility and Its Use in Estimating Stochastic Volatility Models. Royal Statistical 
Society, 64, 253-280.

[8] Zhang, L., Mykland, P.A. and Ait-Sahalia, Y. (2005) A Tale of Two Time Scales: Determining Integrated Volatility with Noisy High-Frequency Data. American Statistical Association, Theory and Methods, 100, 1394-1411.

[9] Large, J. (2007) Estimating Quadratic Variation When Quoted Prices Change by a Constant Increment. University of Oxford, Oxford.

[10] Alvarez, A., Panloup, F. and Savy, N. (2010) Estimation of the Instantaneous Volatility. American Mathematical Society. http://www.arxiv.org/abs/0812.3538v4

[11] Zu, Y. and Boswijk, H.P. (2013) Estimating Spot Volatility with High-Frequency Financial Data. Discussion Paper Series.

[12] Comte, F. and Renault, E. (1998) Long Memory in Continuous Time Stochastic Volatility. Mathematical Finance, 8, 4 291-323.

[13] Foster, D.P. and Nelson, D.B. (1996) Continuous Record Asymptotics for Rolling Sample Variance Estimators. Econometrica, 64, 139-174. https://doi.org/10.2307/2171927

[14] Mykland, P.A. and Zhang, L. (2006) ANOVA for Diffusions and Ito Processes. The Annals of Statistics, 34, 1931-1963.

[15] Andreou, E. and Ghysels, E. (2002) Rolling-Sample Volatility Estimators: Some New Theoretical, Simulation, and Empirical Results. Journal of Business and Economic Statistics, 20, 363-376. http://www.jstor.org/stable/1392123 https://doi.org/10.1198/073500102288618504

[16] He, S., Wang, J. and Yan, J. (1992) Semimartingale Theory and Stochastic Calculus. Science Press, CRC Press Inc.

[17] Billingsley, P. (1995) Probability and Measure. John Wiley and Sons, Hoboken.

[18] Privault, N. (2016) Notes on Stochastic Finance: Stochastic Calculus for Jump Processes. Technical Report, Nanyang Technological University, Singapore. http://www.ntu.edu.sg/home/nprivault/indext.html

[19] Karandikar, R.L. and Rao, B.V. (2007) On Quadratic Variation of Martingales. Proceedings of Indian Academy of Sciences, 124, 3 457-469.

[20] Protter, P.E. (2004) Stochastic Integration and Differential Equations. In: Stochastic Modeling and Applied Probability, 2nd Edition, Springer-Verlag, Berlin, Heidelberg.

[21] Jeanblanc, M. (2007) Jump Processes. Universite d'Evry Val d'Essonne, Cimpa School.

[22] Navas, J.F. (2003) Calculation of Volatility in a Jump-Diffusion Model. The Journal of Derivatives, 11, 66-72. https://doi.org/10.3905/jod.2003.319217

[23] Kanatani, T. (2004) High Frequency Data and Realized Volatility. Ph.D. Thesis, Graduate School of Economics, Kyoto University, Kyoto.

[24] Calderon, C.P. (2009) Lecture III: Review of Classic Quadratic Variation Results and Relevance to Statistical Inference in Finance. PASI, Rice University/Numerica Corporation.

[25] Oksendal, B. (2003) Stochastic Differential Equations: An Introduction with Applications. 6th Edition, Springer-Verlag, New York.

https://doi.org/10.1007/978-3-642-14394-6

[26] AngloGold Ashanti (2011) History. Johannesburg, South Africa. http://www.anglogoldashanti.com/en/About-Us/history/Pages/default.aspx 
Submit or recommend next manuscript to SCIRP and we will provide best service for you:

Accepting pre-submission inquiries through Email, Facebook, LinkedIn, Twitter, etc. A wide selection of journals (inclusive of 9 subjects, more than 200 journals)

Providing 24-hour high-quality service

User-friendly online submission system

Fair and swift peer-review system

Efficient typesetting and proofreading procedure

Display of the result of downloads and visits, as well as the number of cited articles Maximum dissemination of your research work

Submit your manuscript at: http://papersubmission.scirp.org/

Or contact am@scirp.org 